\title{
Luces y sombras del marco normativo del acceso abierto en la Administración General del Estado en España
}

\author{
Néstor CLABO CLEMENTE \\ Centro de Estudios Políticos y Constitucionales \\ nestor.clabo@cepc.es
}

Recibido: Abril 2015

Aceptado: Septiembre 2015

\begin{abstract}
Resumen: El objetivo de esta investigación es identificar cuál es el marco normativo en el que se desenvuelve el acceso abierto en la Administración General del Estado (AGE) de España y cómo condiciona su funcionamiento. Para ello se recopilan exhaustivamente las diversas disposiciones, declaraciones y convocatorias existentes en España y la Unión Europea, y se realiza un análisis comparado de todas ellas. La conclusión es la ausencia de marcos legales comunitarios y nacionales específicos y a la vez de alcance general sobre acceso abierto. Las menciones principales aparecen recogidas de forma parcial o tangencial en programas y convocatorias de financiación de proyectos de investigación, comunicaciones y normativa de reutilización y datos abiertos. Ni siquiera la Ley 14/2011 de la Ciencia, la Tecnología y la Innovación (LCTI) ofrece un mandato suficientemente claro, y la nueva modificación de la Ley de Propiedad Intelectual (LPI) empeora aún más la situación. El tema más tratado es el de los repositorios, lo que indica la preferencia por la vía verde para asegurar el acceso abierto a los contenidos de las revistas.
\end{abstract}

Palabras clave: Acceso abierto; Administración General del Estado; España; Normativa; Política científica; Reutilización; Repositorios; Unión Europea; Vía verde.

\section{The lights and shadows of the open access regulatory framework at the General Government Administration in Spain}

\begin{abstract}
This paper aims at reviewing the open access regulatory frame workat the General Government Administration in Spain to observe how it affects its performance. To achieve such goals this study first compiles and analyses a whole range of rules, provisions, statements and existing calls in Spain and the European Union on comparative bases. The conclusion is the absence of specific and general European and national legal frame work saffecting the open access. The main information collected appear partially ortangentially in programs and calls forfunding of research projects, communications, and reuse and open data regulation. Eventhe Law 14/2011 of Science, Technology and Innovation does not provide a sufficiently clear mandate, and the new amendment to the Copyright Act does not contribute to clarify the situation neither. The focus is usually on the repositories, indicating a preference for the green road to ensure open access to journalcontent.
\end{abstract}


Keywords: Europea Union; General Government Administration; Green Road. Open Access; Regulation; Science Policy; Spain; Reuse; Repositories.

\section{INTRODUCCIÓN}

Muchas son las definiciones que se han proporcionado sobre acceso abierto. A grandes rasgos consiste en el acceso libre, gratuito y sin apenas restricciones de copyright a la literatura científica a través de internet (Budapest Open Access Initiative, 2002; Abadal, 2012; Suber, 2013). Además, el acceso a la información ha de ser inmediato y permanente (Harnad, 2005). Por tanto, entran en juego no sólo aspectos documentales y técnicos, sino también organizativos y legales, que son fundamentales para el desarrollo de las diferentes políticas científicas.

Así, la Federación Internacional de Asociaciones de Bibliotecarios (FIAB) no sólo lo caracteriza como un mero movimiento o tendencia, sino que le añade un carácter institucional al aludir a su necesaria articulación como un modelo organizativo sin el cual las opciones de éxito serán pocas (FIAB, 2011). En este sentido, son importantes las diferentes declaraciones llevadas a cabo por instituciones políticas, académicas y asociaciones a lo largo de estos últimos años (Simmons College, 2014).

En cuanto a la dimensión normativa, son muy importantes los aspectos legales que afectan a la reutilización (Suber, 2013) como parte fundamental del acceso abierto, que queda comprometido si los contenidos publicados de forma gratuita no están libres, entre otras cosas, de las restricciones impuestas por la cesión de los derechos de explotación. Mediante la retención de los mismos por parte del autor y la implementación de adecuadas formas de licenciamiento se asegura la libre difusión y futura reutilización de los contenidos.

Es difícil encontrar gobiernos u organizaciones supranacionales que hayan desarrollado legislación específica acerca del acceso abierto(Caruso, Nicol, \& Archambault, 2013a). La mayoría de la información disponible procede de canales menos normativos, como pueden ser las directrices emanadas de las diversas agencias públicas financiadoras de proyectos de investigación. También es posible rastrearla en otras leyes de reutilización, de copyright o temas similares.

El objetivo de este trabajo es precisamente el análisis de los aspectos legales y organizativos que afectan al acceso abierto aplicado a un ámbito muy específico, como es el de la Administración General del Estado (AGE) ${ }^{1}$, porque es de obligado

${ }^{1}$ La Administración General del Estado está integrada por la Administración Central (Gobierno y Consejo de Ministros, comisiones delegadas del Gobierno, ministerios, Comisión General de Secretarios de Estado y Subsecretarios, comisiones interministeriales), la Administración Periférica (delegaciones del Gobierno en las comunidades autónomas) y la Administración del Estado en el Exterior (embajadas y consulados). Quedan fuera la Administración Autonómica y la Administración Local. 
cumplimiento por parte de todos los actores involucrados en España. Por tanto, se circunscribe a la Unión Europea y a la legislación española de alcance nacional. No se ha descendido hasta el ámbito autonómico o los mandatos de instituciones de investigación como universidades, lo que demandaría un estudio específico de gran envergadura únicamente dedicado a tratar ese tema, y que estimamos que sería muy conveniente para delimitar un mapa de mandatos específicos en todo el país.

Se ha empleado una técnica de investigación cualitativa. Así, se recopila y se comenta con detalle la normativa comunitaria y nacional que afecta directa e indirectamente al desarrollo del acceso abierto en nuestro país. A continuación se establecen comparaciones y se elabora una tabla en la que aparecen de forma expresa todos los aspectos que hay tener en cuenta en el acceso abierto y cómo son afectados o no por las distintas regulaciones o directrices analizadas. Finalmente, se extraen una serie de conclusiones.

\subsection{UNIÓN EUROPEA}

Como hitos más importantes pueden señalarse los siguientes:

\section{Séptimo Programa Marco de Investigación (2007-2013)}

Este Programa incluía un proyecto piloto que prescribía la obligatoriedad de que en siete áreas específicas de investigación los artículos científicos revisados resultado de los proyectos financiados se depositaran en repositorios de acceso abierto.

\section{Digital Repository Infraestructure Vision for European Research (DRIVER)}

También dentro del Séptimo Programa Marco se financió un proyecto de gran importancia, DRIVER, cuyo objetivo era la creación de una infraestructura de servicios y de datos de investigación a partir de la agregación de los contenidos de los repositorios abiertos europeos (Caruso et al., 2013b). DRIVER en el ámbito de la documentación científica desempeñaría un papel similar al de Europeana en relación con el patrimonio documental europeo, de modo que se convirtiera en un portal para mostrar de forma centralizada la producción científica europea de acceso abierto(Toro Sánchez-Blanco, 2009).

\section{Open Access Infrastructure for Research in Europe (OpenAIRE)}

Se trata de un proyecto complementario de DRIVER. El resultado final es la creación de un modelo que promueve el acceso abierto a gran escala (Caruso et al., 2013b). Su objetivo es proporcionar infraestructura tecnológica y organizacional para la identificación, depósito, acceso y monitorización de las publicaciones 
financiadas por el Séptimo Programa Marco y el Consejo Europeo de Investigación (ERC). Por un lado, sirve de apoyo a los investigadores que depositan en repositorios de confianza de los estados miembros; por otro, facilita a los autores sin acceso a repositorios de confianza, el depósito de su trabajo en el OpenAIREOrphan Record Repository. También introduce varios campos que facilitan que en los datos recolectados se especifique la relación del ítem con el proyecto de investigación, el tipo de acceso permitido y la fecha de embargo si la hubiere.

\section{Agenda Digital Europea}

Constituye uno de los siete pilares de la Estrategia 2020 para fijar el crecimiento de la Unión Europea hasta 2020. Su fin primordial es explotar al máximo las posibilidades que las tecnologías de la información y la comunicación nos ofrecen para favorecer el progreso, la innovación y el crecimiento económico. Está estructurada en varias líneas de trabajo. La que más afecta al acceso abierto es aquella que propone el refuerzo de la interoperabilidad y las normas, y que habla expresamente de dispositivos, aplicaciones, repositorios de datos, servicios y redes. También se insiste en la promulgación de regulaciones adaptadas a los derechos de propiedad intelectual, y que en ningún caso entren en contradicción con los nuevos usos que las tecnologías permiten (Comisión Europea, 2010). En 2013, el responsable de la Agenda Digital Europea, NeelieKroes(2013), anunció el lanzamiento de la Research Data Alliance, para la que se requerirá el acceso abierto de todas las publicaciones surgidas al abrigo de proyectos financiados por la Unión Europea. Sin embargo, aún no hay un mandato legal que dé cobertura legal a la declaración.

Tres comunicaciones más de la Comisión Europea han reforzado los principios fundamentales de la Agenda Digital Europea y han marcado las líneas maestras de actuación que han desembocado en los principios conformadores del programa Horizon 2020. La primera, Una asociación del Espacio Europeo de Investigación reforzada en pos de la excelencia y el crecimiento(Comisión Europea, 2012c), insiste en la creación de una zona unificada de investigación en la que investigadores, ciencia y tecnología circulen libremente, y para la que es fundamental el acceso y la transferencia del conocimiento científico (Comisión Europea, 2012c).

Más explícita respecto a las políticas de acceso abierto resultaron la comunicación Hacia un mejor acceso a la información científica: impulsar los beneficios de las inversiones públicas en investigación(Comisión Europea, 2012a), y la recomendación Sobre el acceso y preservación de la información científica(Comisión Europea, 2012b). Ambas inciden en la necesidad de que los estados miembros construyan políticas de acceso abierto basadas en principios similares. 


\section{Horizon 2020}

El Programa Marco de Investigación 2014-2020, denominado Horizon 2020, supone un salto cualitativo muy importante en la implantación de políticas claras de acceso abierto en la Unión Europea. Su principio básico es rentabilizar las inversiones públicas en investigación financiadas por este programa, de modo que no se pague dos veces por ellas: una para financiarlas y otra para el acceso y el uso de la información producto de los resultados de las investigaciones realizadas (Comisión Europea, 2013). Se establece como beneficiarios principales a las empresas europeas y a los ciudadanos. Así, la información debe ser puesta a disposición de investigadores europeos, industrias innovadoras y ciudadanos, siempre online y de modo gratuito. A su vez, tan importante como el acceso es la preservación a largo plazo, algo que resulta novedoso en este programa marco frente al anterior, y que sin duda incide en la dimensión de conservación futura de la información contenida en los repositorios.

Horizon 2020 establece unas reglas claras de participación. Así, todos los beneficiarios deben asegurar el acceso abierto a todos los artículos arbitrados relacionados con los resultados de la investigación. También es significativo que se incluyan otras tipologías documentales, de modo que se anima a los investigadores a que, dentro de lo posible, proporcionen acceso a monografías, comunicaciones, informes, ponencias, etc.

En cuanto a la forma de depósito, los requisitos son bastante claros: los autores deben depositar una copia electrónica de los artículos en repositorios y siempre en formatos interoperables, independientemente de que el artículo en cuestión haya sido publicado en abierto en alguna revista mediante la vía dorada o mediante la modalidad de publicación hibrida. También se fijan plazos, de modo que la fecha límite debe ser la de publicación en la revistas. Respecto a las versiones, Horizon 2020 permite el depósito de cualquier versión, aunque se recomienda fuertemente que sea la publicada.

También se dan directrices para la elección de los repositorios adecuados. Por ello, se recomienda el acceso a la plataforma OpenAIRE, que servirá como punto de referencia para la elección del repositorio más adecuado, aunque tampoco se descartan otras fuentes de referencia como ROAR (Registry of Open Access Repositories) u OpenDOAR. Se establece como plazo máximo de acceso los seis meses cuando haya algún tipo de embargo que así lo estipule. Incluso, cuando para facilitar el acceso el autor tenga que pagar tasas, como ocurre bastantes veces con revistas que siguen el modelo dorado o el híbrido, se contempla la posibilidad de un reembolso que cubra los gastos generados.

Sin duda, una de las grandes novedades hay que buscarla en la insistencia que Horizon 2020 pone en los datos de investigación o, dicho de otra forma, los datos anejos a la misma, cuya explotación da lugar a sus resultados. En esta política puede verse claramente la confluencia de los movimientos a favor de los datos 
abiertos y del acceso abierto, las dos caras de la misma moneda. Es crucial la introducción de un programa piloto, el Open Research Data Pilot, para mejorar y potenciar el acceso y reutilización de los datos usados en el proyecto, y que afecta a unas áreas prioritarias. Dichos proyectos deben incluir un data management plan, que recoge detalladamente qué tipos de datos se van a recolectar, cómo, qué estándares, formatos y qué acceso se va dar a los mismos.

Otro aspecto en el que se incide especialmente en Horizon 2020 es la normalización. Así, se indica lo necesario de adjuntar metadatos descriptivos, incluidos los del proyecto concedido. También se recomienda el uso de identificadores: DOIS para artículos científicos, DataCite para datos y ORCID (Open Researcher and ContributorID) para investigadores.

Por último, también se anima a los autores a conservar el copyright de sus artículos, especialmente mediante licencias CC-By y CC-0. Este aspecto del uso de licencias se está convirtiendo en algo crucial en las políticas de acceso abierto y de datos abiertos.

\section{Directiva 2003/98/CE relativa a la reutilización de la información en el sector público}

La reutilización es una de las modalidades principales que adopta el acceso abierto de las publicaciones, desde la comunicación pública de parte de sus contenidos y la copia, hasta la explotación comercial y la mezcla y creación de obras derivadas

La norma comunitaria incluye sin distinción todos los documentos difundidos por organismos públicos, y prescribe que puedan reutilizarse para fines comerciales y no comerciales, salvo que estén sujetos a restricciones derivadas de la normativa de acceso, tal y como ya recoge España en el Real Decreto 1495/2011, de 24 de octubre, de desarrollo de la Ley 37/2007 de 16 de noviembre, sobre reutilización de la información del sector público para el ámbito del sector público estatal (RDRISP). También se establece la obligación de ofrecer los datos en formatos legibles mediante máquinas para garantizar su efectiva reutilización.

Dicha directiva ha sido sustituida por otra, la 2013/37 UE del Parlamento Europeo y del Consejo de 26 de junio de 2013. Uno de los aspectos más novedosos es la clarificación del ámbito de aplicación, por lo que no se aplicará a documentos no relacionados con la actividad administrativa o a aquellos que entren de lleno en el terreno de la confidencialidad comercial (Gallo, 2013). Por tanto, cabe pensar que no es aplicable a los artículos de las revistas científicas. Sin embargo, en la normativa española de reutilización, aunque en primer término también se había aplicado dicha distinción, posteriormente el RDRISP da marcha atrás, por lo que las normas de reutilización se deberían aplicar a los datos y el resto de documentos generados por la Administración, entre los que se encuentran no sólo los meramente administrativos, sino también las publicaciones financiada 
públicamente cuyos derechos de explotación le han sido cedidos. Este es, sin duda, un aspecto muy importante.

\section{LEGISLACIÓN ESPAÑOLA}

\subsection{LEY 37/2007 SOBRE REUTILIZACIÓN DE LA INFORMACIÓN DEL SECTOR PÚBLICO (LRISP)}

Esta ley obliga también a las publicaciones financiadas públicamente. De ahí, la importancia de este tema y cómo lo trata la normativa española. Incluso aquellos contenidos afectados por derechos de propiedad intelectual, con el Real Decreto de reutilización de la información del sector público (RDRISP) también acaban cayendo dentro de su ámbito de aplicación. Desde luego, la tesis que se mantiene en este trabajo es que los contenidos publicados en revistas científicas financiadas por la AGE, sean o no resultado de otros proyectos de investigación también financiados públicamente, deben cumplir el mandato de reutilización contenido en la LRISP y el RDRISP.

La adaptación al ordenamiento jurídico español de la Directiva 2003/98/CE se produce mediante la LRISP, que extiende el marco de aplicación a todas las administraciones públicas. La normativa española incluye toda la tipología documental posible, desde los datos primarios y secundarios hasta todo tipo de publicaciones, independientemente de su forma de expresión, aunque por su contenido quedan excluidos los afectados por derechos de propiedad intelectual. Parece, por tanto, que quedarían fuera gran parte de los contenidos generados por investigadores o grupos de investigación, independientemente de que hayan sido financiados con fondos públicos. Sin embargo, con posteridad el RDRISP incluye dentro del ámbito de la reutilización también los documentos sujetos a derechos de propiedad intelectual cuyos derechos de explotación hayan sido cedidos por parte de sus titulares. Esta rectificación incluye dentro del ámbito de aplicación de la norma a las publicaciones científicas editadas por la AGE y, por tanto, a sus revistas científicas (Clabo y Ramos, 2015).

También trata otros temas que afectan directamente a las publicaciones científicas, como son la posibilidad del cobro de tasas y precios públicos, el procedimiento de tramitación de solicitudes de reutilización y el régimen sancionador. Sin embargo, los aspectos más interesantes son el establecimiento de modalidades de puesta a disposición, las condiciones que deben cumplir las licencias y la prohibición de acuerdos exclusivos.

Por último, conviene señalar un aspecto que nos ofrece dudas en cuanto a la difusión en acceso abierto de la ciencia. Se trata de que los documentos producidos o conservados por instituciones de investigación, incluidas las 
organizaciones para la transferencia de resultados, quedan explícitamente fuera del ámbito de aplicación de esta ley.

\subsection{REAL DECRETO 1495/2011 POR EL QUE SE DESARROLLA LA LEY 37/2007}

El RDRISP no sólo recoge los preceptos de la LRISP, sino que los desarrolla teniendo en cuenta también las diferentes aportaciones internacionales surgidas tras ese año. Así, en el preámbulo se cita el Plan de Acción de la Unión Europea sobre Administración Electrónica para el período 2011-2015, la Declaración Ministerial de Granada de abril de 2010 y la nueva Agenda Digital Europea de mayo de 2010, ya comentada. También se enmarca en el conjunto de medidas que constituyen la Estrategia 2011-2015 del Plan Avanza2 (Clabo y Ramos, 2015).

En cuanto a acceso abierto lo más interesante es que fija las condiciones de reutilización, de modo que pone a disposición de los ciudadanos la información sin condiciones específicas, salvo los límites marcados por la propiedad intelectual e industrial de terceros. Para ello, incluye un anexo con un aviso legal que recomienda usar en las páginas web de la AGE.

\subsection{MODALIDADES DE REUTILIZACIÓN EN LA NORMATIVA ESPAÑOLA}

El artículo 4 de la LRISP, incluido en el Título II, referido al régimen jurídico de la reutilización, permite tres modalidades de reutilización. De ellas, las dos primeras son las que permiten un uso libre o licenciado:

- Reutilización sin sujeción a ningún tipo de condiciones. La información puesta a disposición de esta manera podrá ser reutilizada sin ningún tipo de condición, por lo que no deberá acogerse a ningún tipo de licencia. Sin embargo, el RDRISP incluye en su Anexo un aviso legal que tiene alcance general para todo tipo de documentos de la AGE susceptibles de ser reutilizados siempre y cuando no estén sujetos a otro tipo de licencia, y que preferentemente debe aplicarse a los documentos tratados en este apartado. Por tanto, puede afirmarse que es una licencia por defecto compatible con la definición de "conocimiento abierto" establecida por la Open Knowledge Foundation(Ramos Simón et al., 2012). En él se enumeran las modalidades de reutilización, que son las siguientes: copia, difusión, modificación, adaptación, extracción, reordenación y combinación de la información. Curiosamente, desaparece una de las modalidades de la LRIS contenida en su artículo 8, en concreto la que prohibía que el contenido de la información fuera alterado. De nuevo, aquí el legislador de 2011 parece corregir al de 
2007, y lo realiza con buen criterio, ya que el contenido de la información puede ser alterado siempre que así se declare y esté permitido por las licencias de uso.

- En el caso de que la Administración opte por establecer condiciones, recomienda el uso de licencias-tipo utilizadas hoy en día en el ámbito de la normativa de propiedad intelectual. El RDRISP va un paso más allá y en su artículo 8.3 establece explícitamente que podrán emplearse licencias-tipo existentes, denominadas "libres", siempre que se ajusten a lo establecido en el real decreto. Nada se dice sobre licencias CC y Open Data Commons, pero parece que la alusión es innegable.

- Reutilización de documentos previa solicitud, conforme al procedimiento previsto en el artículo 10 o, en su caso, en la normativa autonómica, pudiendo incorporar también en estos supuestos condiciones establecidas en una licencia.

Respecto a la titularidad de los documentos susceptibles de reutilización, la LRISP en su artículo 3, referido al ámbito de aplicación de la ley, hace una diferenciación fundamental respecto a la tipología documental, que se va a mantener tanto a lo largo de esta norma como del RDRISP, donde se desarrolla extensamente. Se trata de la titularidad de los derechos de propiedad intelectual. Así, distingue entre dos tipos de documentos:

- Documentos sujetos a propiedad intelectual o industrial por parte de terceros, que quedan excluidos del alcance de la ley.

- Documentos sujetos a propiedad intelectual por parte de las Administraciones y organismos del sector público, que sí entran dentro del ámbito de aplicación de la ley.

Esta primera división no era muy acertada, ya que dejaba fuera de la esfera de la reutilización muchos documentos de autor, bien sean personales o institucionales ajenos a la Administración, sujetos a propiedad intelectual y que, sin embargo, son editados por organismos públicos que poseen los derechos de explotación cedidos legalmente. Dado que la reutilización incluye una serie de modalidades como son la copia y difusión, estos documentos entrarían de lleno también el ámbito de la LRISP. Curiosamente, el RDRISP parece que advierte el olvido previo del legislador y en su artículo nueve incluye dentro del ámbito de la reutilización también los documentos sujetos a derechos de propiedad intelectual cuyos derechos de explotación hayan sido cedidos por parte de sus titulares. Esta rectificación incluye dentro del ámbito de aplicación de la norma a las publicaciones científicas editadas por la AGE y, por tanto, a sus revistas científicas.

Por último, también hay que señalar que se permite el uso comercial y el no comercial de la obra o documento derivado, y este es una de las claves de la norma, ya 
que una de las principales razones para justificarla, como ya expresaba con toda claridad la Directiva 2003/98/CE, era la rentabilidad económica emanada de la posible reutilización de los documentos del sector público, algo que también señala Horizon 2020 al hablar del acceso y utilización de los resultados de las investigaciones.

\subsection{LEY 18/2015POR LA QUE SE MODIFICA LA LRISP}

Esta ley de modificación tiene su razón de ser en la incorporación al ordenamiento jurídico español de los cambios en el régimen de reutilización de documentos del sector público introducidos por la Directiva 2013/37/UE del Parlamento Europeo y el Consejo, de 26 de junio de 2013.

Introduce aspectos positivos para el acceso abierto al promover explícitamente el uso de formatos abiertos y legibles por máquina junto con sus metadatos, así como el cumplimiento de las normas de interoperabilidad. También es importante la obligación establecida de promover el uso de licencias abiertas, de tal forma que para la reutilización de la información del sector público se planteen las mínimas restricciones posibles.

Otra modificación reseñable, que se hace para recoger los preceptos emanados del Plan Estatal de Investigación Científica, Técnica y de Innovación 2013-2016, es la acotación que se hace de los resultados producidos por instituciones públicas de investigación, que si en la LRISP quedaban fuera del ámbito de la reutilización, ahora no lo hacen en un sector específico: los microdatos generados en encuestas correspondientes a investigaciones de ciencias sociales, que un plazo de cuatro años, y debidamente anonimizados, deben ser depositados en el Banco de Datos del Centro de Investigaciones Sociológicas. Esta obligación también se hace extensible al sector privado siempre y cuando el presupuesto para sus proyectos de investigación proceda, al menos, en un $50 \%$ de financiación pública. Cabe preguntarse si esta obligación no podría haberse planteado en otras disciplinas académicas, que igualmente cuentan con financiación pública.

\subsection{LEY 14/2011 DE LA CIENCIA, LA TECNOLOGÍA Y LA INNOVACIÓN}

En España, la única norma que alude directamente al acceso abierto es la LCTI. Lo hace en el Preámbulo como declaración de principios de alcance general y ya más detalladamente en su artículo 37. Sin embargo, no lo hace de forma muy decidida y deja muchos interrogantes sin resolver.

En dicho artículo afirma que "los agentes públicos del Sistema Español de Ciencia, Tecnología e Innovación impulsarán el desarrollo de repositorios, propios o compartidos, de acceso abierto a las publicaciones de su personal de investigación, y establecerán sistemas que permitan conectarlos con iniciativas similares de ámbito nacional e internacional” (España. Jefatura del Estado, 2011: 54425). 
A continuación establece que el personal investigador cuya actividad investigadora esté financiada mayoritariamente con fondos de los Presupuestos Generales del Estado debe hacer pública la versión final de los contenidos de la investigación aceptados en publicaciones periódicas o seriadas tan pronto como sea posible, fijando el máximo de tiempo que puede discurrir en 12 meses. Aquí cabe comentar dos aspectos que inciden en la tibieza de la medida. Lo primero es la precisión deliberada que se hace en cuanto a la cuantía de la participación de los fondos públicos: sólo se establece el mandato de poner a disposición del público en general los contenidos de la investigación cuando la financiación de la Administración es mayoritaria. ¿Qué ocurre cuando así no sea, incluso cuando hablamos de una participación casi paritaria que pueda rondar el 50\%? Pero incluso aunque no alcance ese nivel, no parece muy razonable que proyectos financiados públicamente, aunque no lo sean en su totalidad ni siquiera mayoritariamente, estén exentos de la obligatoriedad de hacerse públicos en acceso abierto.

El segundo aspecto que ha generado gran controversia es la fijación del plazo máximo en 12 meses desde su publicación en revistas científicas. Parece desproporcionado y choca con los plazos definidos por Horizon 2020, que situaba en seis meses el período de embargo. Hasta hace relativamente poco había un consenso tácito entre la comunidad científica de que únicamente las humanidades y las ciencias sociales podrían acogerse a un plazo de ese tipo, mientras que para el resto de disciplinas se situaría en seis. Sin embargo, hoy día, con el desarrollo de las tecnologías de la comunicación y la información y el aumento de la rapidez en el acceso, la difusión y puesta a disposición de contenidos a través de las redes sociales, no parece muy defendible la diferencia que pueda establecerse entre disciplinas. Tampoco va bien encaminada la medida en el sentido en que las tendencias internacionales de acceso abierto tienden a acortar plazos de forma drástica, de modo que hay cierto acuerdo entre las previsiones de los máximos especialistas en la materia, que anticipan un gran incremento de la vía dorada y un acortamiento notable de los plazos contemplados en la vía verde (Neylon, 2013;Rizor y Holley, 2014;Working Group on Expanding Access to Public Research Findings, 2012).

El último punto que genera gran controversia, por su ambigua redacción, es el que señala que "lo anterior se entiende sin perjuicio de los acuerdos en virtud de los cuales se hayan podido atribuir o transferir a terceros los derechos sobre las publicaciones, y no será de aplicación cuando los derechos sobre los resultados de la actividad de investigación, desarrollo e innovación sean susceptibles de protección” (España. Jefatura del Estado, 2011: 54425). Su lectura genera muchas dudas: ¿se entiende que la obligatoriedad de poner en acceso abierto las investigaciones queda en suspenso si se han transferido a terceros los derechos de explotación? Si es así, este punto genera una inseguridad jurídica que deja en suspenso cualquier medida tomada en esta ley en favor del acceso abierto. La política editorial de una revista no puede estar por encima ni fijar los requisitos de 
acceso y embargo de artículos afectados por el ámbito de aplicación de la LCTI. Cualquier sombra que se proyecte sobre ese principio es muy nociva, y este punto es justamente lo que hace. Llevado a sus últimas consecuencias, si un grupo de investigación publicara sus resultados en una revista que exigiera la retención en exclusiva de todos los derechos de explotación, no tendrían la obligación ni de publicar en abierto los resultados, aun pasado un período de embargo, ni de depositarlos en un repositorio. Como se ve, las consecuencias de esta mala redacción pueden ser muy negativas.

\subsection{PLAN ESTATAL DE INVESTIGACIÓN CIENTÍFICA, TÉCNICA Y DE INNOVACIÓN 2013-2016}

El Plan cuenta con once objetivos específicos. El décimo es el que afecta al tema que se está investigando. En concreto, busca "incrementar la cultura científica, tecnológica e innovadora de la sociedad española, así como la difusión de los resultados de la investigación científico-técnica y de la innovación financiados con fondos públicos” (España. Ministerio de Economía y Competitividad, 2013: 11). Sin embargo, no hace alusión alguna a ningún mandato específico para el cumplimiento de este objetivo. Tampoco menciona en ningún momento el acceso abierto, lo cual resulta bastante llamativo en un documento de este tipo.

Este Programa se concreta anualmente en convocatorias de procedimientos de concesión de ayudas. La última ha sido aprobada mediante resolución de 17 de junio de 2015(España. Secretaría de Estado de Investigación, Desarrollo e Innovación, 2015). Ahí sí se pueden encontrar dos referencias. La primera, incluida en el artículo 6, recuerda la obligación del cumplimiento de lo estipulado en el artículo 37 de la LCTI, pero recorta el plazo de publicación en acceso abierto o el autoarchivo en repositorios institucionales en seis meses, excepto para las investigaciones de ciencias sociales y humanidades, que lo mantienen en doce.

La segunda se encuentra en el artículo 8.2. Allí se dice que los proyectos que impliquen la realización de estudios mediante encuestas cuantitativas en el ámbito de las ciencias sociales con toma de datos, deberán cumplir con lo establecido en la LRISP y la recomendación de la Comisión Europea de 17 de julio de 2012, relativa a la información científica y su preservación, de la que ya se ha hablado. En la actualidad, el centro de depósito es el Banco de Datos Específico de Ciencias Sociales (ARCES), gestionado por el Centro de Investigaciones Sociológicas y que pretende convertirse en un repositorio institucional destinado a conservar y difundir los datos de investigación producidos dentro de proyectos y trabajos realizados con cargo a los presupuestos de las Administraciones Públicas. 


\section{RESULTADOS COMPARADOS DEL ANÁLISIS NORMATIVO}

Tras el análisis, se ha corroborado que no hay normativa tanto a nivel comunitario como español que trate en exclusiva el tema del acceso abierto. La mención al mismo y sus temas relacionados aparecen recogidos fundamentalmente en programas y convocatorias de financiación de proyectos de I+D, comunicaciones y declaraciones de alcance general y normativa de reutilización y datos abiertos, y casi siempre de forma parcial o tangencial.

Tabla 1. Normas y programas en España y la UE que recogen información sobre acceso abierto y temas afines

\begin{tabular}{|c|c|c|c|c|c|c|c|c|c|c|c|c|}
\hline & \multirow[b]{2}{*}{$\begin{array}{c}\text { Mención } \\
\text { "acceso } \\
\text { abierto" }\end{array}$} & \multirow[b]{2}{*}{$\begin{array}{l}\text { Reposito- } \\
\text { rios }\end{array}$} & \multirow[b]{2}{*}{ Versiones } & \multirow[b]{2}{*}{ Embargo } & \multirow[b]{2}{*}{$\begin{array}{c}\text { Preserva- } \\
\text { ción }\end{array}$} & \multirow[b]{2}{*}{$\begin{array}{c}\text { Incluye } \\
\text { datos }\end{array}$} & \multicolumn{2}{|c|}{ Reutilización } & \multicolumn{3}{|c|}{ Normas } & \multirow[b]{2}{*}{ Total } \\
\hline & & & & & & & $\begin{array}{c}\text { Reutiliza- } \\
\text { ción }\end{array}$ & Licencias & $\begin{array}{c}\text { Interopera- } \\
\text { bilidad }\end{array}$ & $\begin{array}{c}\text { Metada- } \\
\text { tos }\end{array}$ & DOIs & \\
\hline 70 Programa Marco & & $\mathrm{x}$ & & & & & & & & & & 1 \\
\hline DRIVER & & $\mathrm{x}$ & & & & & & & & & & 1 \\
\hline OpenAIRE & & $x$ & & & & & & & $x$ & $\mathbf{x}$ & & 3 \\
\hline Agenda Digital Europea & $\mathbf{x}$ & $\mathbf{x}$ & & & & & & & $\mathbf{x}$ & & & 3 \\
\hline COM (2012) 392 Final & $x$ & & & & & & & & & & & 1 \\
\hline $\operatorname{COM}(2012) 401$ Final & $\mathbf{x}$ & & & & & & & & & & & 1 \\
\hline$C(2012) 4890$ Final & & $x$ & & & & & & & & & & 1 \\
\hline Horizon 2020 & $x$ & $x$ & $x$ & 6 meses & $x$ & $x$ & & $x$ & & $\mathrm{x}$ & $x$ & 9 \\
\hline Directiva 2003/98/CE & & & & & & $\mathbf{x}$ & $\mathbf{x}$ & & & & & 2 \\
\hline LRISP & & & & & & $\mathrm{x}$ & $x$ & $\mathrm{x}$ & & & & 3 \\
\hline RDRISP & & & & & & $x$ & $x$ & $x$ & & & & 3 \\
\hline LCTI & $x$ & $x$ & & 12 meses & & & & & & & & 3 \\
\hline Plan Estatal Investigación & & & & & & & & & & & & 0 \\
\hline Total & 5 & 7 & 1 & 2 & 1 & 4 & 3 & 3 & 2 & 2 & 1 & \\
\hline
\end{tabular}

Fuente: elaboración propia

Como puede verse en la tabla 1 , son pocas también las normas o convocatorias que de forma integrada aglutinan los principales temas que han de suscitarse cuando se habla de acceso abierto. La excepción lo constituye el Programa Marco Horizon 2020. Sin duda, la mirada que ofrece es la más completa y abarca casi en su totalidad todos los aspectos de interés: repositorios, versiones, período de embargo, datos anejos, preservación y otros relacionados con la reutilización y normas como las licencias de uso, los metadatos y el empleo de DOIS.

El tema que más veces aparece recogido es el referido a los repositorios. Figura de forma destacada en los diferentes programas marco de la Unión Europea, tanto en el Séptimo como en Horizon 2020. También es explícita su presencia en la Agenda Digital Europea y, en nuestro ámbito, en la LCTI. Esto indica que tanto en el espacio comunitario como en el nacional la política que parece que mejor asegura el acceso abierto es la vía verde frente a la via dorada. Se entiende que el acceso y la preservación de los contenidos quedan mejor garantizados si la 
responsabilidad cae del lado de repositorios institucionales y especializados y no del lado de las revistas, sujetas a diferentes políticas editoriales, y que por definición son contingentes. Sin embargo, también es destacable que Horizon 2020 de algún modo también promociona esta última vía al ofrecer financiación para hacer accesibles de modo gratuito también publicaciones en revistas que siguen el modelo dorado o el híbrido.

Los dos grandes proyectos que impulsan una política regional de repositorios son DRIVER y OpenAIRE. El objetivo del primero es crear una infraestructura de servicios y de datos de investigación a partir de la agregación de los contenidos de los repositorios abiertos europeos, mientras que el segundo ofrece infraestructura tecnológica y organizacional para la identificación, depósito, acceso y monitorización de las publicaciones financiadas por los programas marco.

Dese el punto de vista de la AGE, que es el ámbito de esta investigación, es muy llamativo que la única mención explícita aparezca en la LCTI, y únicamente de modo breve en el preámbulo y el artículo 37. Además, como se ha comentado, arroja dudas sobra la obligatoriedad de depositar en acceso abierto los contenidos si sobre los mismos pesa la cesión de los derechos de explotación a terceros. Tampoco resulta muy alentador que fije en 12 meses el período máximo de embargo, lo que le sitúa fuera de sintonía con Horizon 2020. En la misma línea se sitúa el Plan Estatal de Investigación 2013-2016, donde en ningún momento se menciona el acceso abierto. Para encontrar algo hay que irse a las convocatorias anuales de procedimientos de concesión de ayudas, en las que se refiere a lo estipulado en la LCTI.

En cuanto a la obligatoriedad de difundir en acceso abierto, afecta a los investigadores cuya actividad está financiada mayoritariamente con fondos públicos. "Este requerimiento no debería estar limitado a los proyectos de los Planes Estatales, sino que se podría ampliar a cualquier actividad de investigación y hacerlo por defecto a todo el personal del sistema público de investigación [...]" (FECYT, 2014: 17-18).

Desde el punto de vista cuantitativo, el tema al que se presta mayor atención en nuestro país es la reutilización. La principal peculiaridad, y por lo cual se le ha conferido importancia en esta investigación, es que no afecta únicamente a los datos. Se debe a que la tipología documental abarcada por la LRISP y la RDRISP es muy amplia. Incluso incluyen aquellos que están afectados por derechos de propiedad intelectual cuyos titulares hayan cedido a la Administración la cesión de los derechos de explotación. Por tanto, es conveniente que los contenidos publicados en revistas científicas financiadas por la AGE, sean o no resultado de proyectos de investigación también financiados públicamente, deben cumplir el mandato de reutilización contenido en la LRISP y el RDRISP.

En cuanto a las modalidades de reutilización, también quedan fijadas mediante la LRISP y el RDRISP, que establecen un uso libre o licenciado, y se estima que son aplicables al ámbito de las revistas científicas. 


\section{CODA: LA NUEVA LEY DE PROPIEDAD INTELECTUAL}

Mientras se estaba finalizando este trabajo de investigación, ha entrado en vigor la Ley 21/2014, de 4 de noviembre, por la que se modifica el texto refundido de la Ley de Propiedad Intelectual, aprobado por Real Decreto Legislativo 11996, de 12 de abril, y la Ley 1/2000, de 7 de enero, de Enjuiciamiento Civil(LPI). Se ha levantado mucha polvareda y las implicaciones son grandes, aunque también hay cierta confusión al respecto.

El primer aspecto que influye directamente en el acceso abierto es el carácter irrenunciable al cobro de compensación por copia privada (España. Jefatura del Estado, 2014: artículo 25.2), incluso en los casos en los que no se necesite autorización previa del autor, entre los que se incluye aquellas cuya reproducción esté autorizada por su titular (España. Jefatura del Estado, 2014: artículo 31.2). En este último caso cabe interpretar que se encuentran las obras licenciadas. Como poco, es contradictorio definir como "irrenunciable" un derecho que pertenece originalmente a los autores, que tienen la potestad de decidir qué quieren hacer con él: cobrar, no cobrar, cederlo a las editoriales, licenciarlo, etc.

De cualquier modo, la cuantía y consignación de la compensación se hará con cargo a los Presupuestos Generales del Estado. ¿Cómo se calculará? Esa ya es una cuestión que aquí no se puede contestar, pero lo realmente importante es que el concepto de "copia privada", aunque sea para fines meramente docentes o de investigación y su uso esté licenciado, exige compensación económica.

Si la nueva situación creada no fuera lo suficientemente grave, empeora aún más con el derecho de cita en el ámbito universitario (España. Jefatura del Estado, 2014: artículo 32.3), ya que se establece que los centros han de compensar directamente a los autores citados cuando se produzca un acto de reproducción, distribución o comunicación pública de pequeños fragmentos de obras o capítulos de libros y artículos de revistas. Resulta realmente chocante, no sólo para las raquíticas arcas de las universidades españolas, sino también para el impacto negativo que puede tener en la citación de los autores, y más aún si cabe cuando estos han podido licenciar sus contenidos para que puedan ser reproducidos libremente. También es criticable la equiparación que se da entre capítulos de libros y artículos de publicaciones periódicas, como si fueran entidades equivalentes.

De lo dicho hasta ahora, se deduce el impacto negativo que estas medidas puede tener en las políticas de acceso abierto, ya que restringen y penalizan la difusión de las investigaciones, pero el citado artículo 32 aún puede tener otro efecto adicional no deseado para los repositorios y, por consiguiente, para la vía verde de acceso a la ciencia. ¿Pueden ser asimilados a los "prestadores de servicios electrónicos de agregación de contenidos de fragmentos no significativos divulgados en publicaciones periódicas” recogidos en la Ley? Pues aunque el texto es confuso, aparentemente sí, ya que recopilan documentos y ofrecen información básica que suele incluir resúmenes extraídos de las publicaciones originales. Sin embargo, 
algunos expertos creen que únicamente pueden ser considerados "prestadores de servicios” aquellos que desarrollan una actividad económica (Adsuara, 2014). Por tanto, desde este punto de vista no sería el caso de los repositorios, pero la confusión es grande y, de cualquier modo, el canon por cita y el derecho a compensación ya comentados afectan también muy directamente a los repositorios, ya que contribuyen a restringir económicamente los derechos de copia, difusión, reutilización y comunicación pública de gran parte de los artículos allí contenidos.

Corresponde a expertos legales en la materia decidir si esta modificación de la LPI del 96 atenta contra los principios contenidos en la LCTI y la LRISP y RDRISP, pero lo que sí puede adelantarse es que perjudican claramente los principios básicos que rigen el comportamiento de la ciencia en acceso abierto.

\section{CONCLUSIONES}

El marco normativo que afecta al acceso abierto en España tiene dos ámbitos: el comunitario y el nacional. Tanto en uno como en otro se manifiesta la ausencia de una norma de alcance general. Las menciones principales aparecen recogidas de forma parcial o tangencial en programas y convocatorias de financiación de proyectos de investigación, comunicaciones, declaraciones de alcance general y normativa de reutilización y datos abiertos.

El tema que más veces aparece recogido es el referido a los repositorios. Figura de forma destacada en los diferentes programas marco de la Unión Europea, la Agenda Digital Europea y en nuestra LCTI. Esto indica el predomino de la vía verde frente a la vía dorada y parece indicar que el acceso y la preservación de los contenidos quedan mejor garantizados si la responsabilidad cae del lado de repositorios institucionales y especializados y no del lado de las revistas.

En el ámbito comunitario, el Programa Marco Horizon 2020 es la fuente principal para la regulación del acceso abierto. Comprende gran parte de los aspectos que hay que tener en cuenta: repositorios, versiones de autoarchivo, período de embargo, datos anejos, preservación y otros relacionados con la reutilización y normas como las licencias de uso, los metadatos y el empleo de DOIS. También ofrece financiación para hacer accesibles de modo gratuito publicaciones en revistas que siguen los modelos dorado e híbrido.

En el ámbito nacional, aún a sabiendas de que puede haber otras opiniones, nuestra conclusión es que la situación del mandato legal sobre acceso abierto no es todo lo buena que cabría desear. La única mención explícita aparece en la LCTI y arroja algunas dudas sobre la obligatoriedad de depositar ciertos contenidos en acceso abierto. También fija una política de embargo demasiado permisiva, en clara falta de sintonía con Horizon 2020. Tampoco resulta muy alentadora la ausencia total de mención en el Plan Estatal de Investigación 2013-2016. Para encontrar algo hay que irse a las convocatorias anuales de procedimientos de concesión de ayudas, en las que se refiere a lo estipulado en la LCTI. 
Desde el punto de vista cuantitativo, el tema al que se presta mayor atención en España es la reutilización. La normativa al respecto, recogida en la LRISP y el RDRISP, no afecta sólo a los datos, sino también a las publicaciones, dentro de las cuales hay que incluir las revistas científicas financiadas públicamente, que deben cumplir, por tanto, el mandato legal y las modalidades de reutilización.

También creemos que la reciente modificación de la LPI puede interferir el funcionamiento del acceso abierto en la ciencia: conceptos como la compensación por copia privada, el derecho de cita en el ámbito universitario y la asimilación de los repositorios a prestadores de servicios electrónicos de agregación de contenidos de fragmentos no significativos divulgados en publicaciones periódicas, lejos de disipar dudas y contribuir a mejorar el acceso, visibilidad y reutilización de la producción científica, son susceptibles de entrar en contradicción con los principios contenidos en la LCTI, la LRISP y la RDRISP.

Por último, queremos señalar que nuestra intención es que el análisis, los datos y las opiniones vertidas en este artículo, puedan servir como documento de discusión para que diferentes y hasta opuestos análisis y propuestas a las aquí expuestas, puedan enriquecer el debate y servir de ayuda y desarrollo al ulterior marco normativo del acceso abierto en nuestro país.

\section{REFERENCIAS BIBIOGRÁFICAS}

ABADAL, E. (2012). Acceso abierto a la ciencia Ernest Abadal [consulta: 2-102014]. Disponible en: http://diposit.ub.edu/dspace/bitstream/2445/24542/6/ 262142.pdf.txt

ADSUARA, B. (2014). “Canon AEDE: vayamos por partes”. LawyerPress [blog], 20-7-2014 [consulta: 4-1-2015]. Disponible en: http://www.lawyerpress.com/news/2014_07/3007_14_006.html

BUDAPEST Open Access Initiative (2002). [Consulta: 28-9-2014]. Disponible en: http://www.budapestopenaccessinitiative.org/read

CARUSO, J.; Nicol, A. y Archambault, E. (2013a). Open Access Strategies in the European Research Area. Montreal: Science-Metrix [consulta: 6-10-2014]. Disponible en: http://www.science-metrix.com/pdf/SM_EC_OA_Policies.pdf

CARUSO, J.; Nicol, A. y Archambault, E. (2013b). Open Data Access Policies and Strategies in the European Research Area and Beyond [consulta: 10-102014]. Montreal: Science-Metrix [consulta: 4-11-2014]. Disponible en: http://www.science-metrix.com/pdf/SM_EC_OA_Data.pdf

CLABO, N.; Ramos-Vielba, I. (2015). "Reutilización de datos abiertos en la Administración Pública en España y uso de licencias-tipo". Revista Española de Documentación Científica, 38 (3) [próximamente]

COMISIÓN EUROPEA (2010). Agenda Digital Europea [consulta: 4-2-2015]. Disponible en: http://eur-lex.europa.eu/legal-content/ES/TXT/PDF/?uri= CELEX:52010DC0245\&from=es 
COMISIÓN EUROPEA (2012a). Hacia un mejor acceso a la información científica: impulsar los beneficios de las inversiones públicas en investigación[consulta: 23-11-2014]. Disponible en:http://eurlex.europa.eu/LexUriServ/LexUriServ.do?uri=COM:2012:0401:FIN:ES:PDF

COMISIÓN EUROPEA (2012b). Recomendation of 17.7.2012 on access to and preservation of scientific information [consulta: 12-12-2015]. Disponible en: http://ec.europa.eu/research/sciencesociety/document_library/pdf_06/recommendation-access-and-preservationscientific-information_en.pdf

COMISIÓN EUROPEA (2012c). Una asociación del Espacio Europeo de Investigación reforzada en pos de la excelencia y el crecimiento [consulta: 1312-2015]. Disponible en: http://eur-lex.europa.eu/LexUriServ/LexUriServ.do? uri=COM:2012:0392:FIN:ES:PDF

COMISIÓN EUROPEA (2013). Guidelines on Open Access to Scientific Publications and Research Data in Horizon 2020. Version 1.0 [consulta: 3-12015]. Disponible en: http://ec.europa.eu/research/participants/data/ref/h2020/ grants_manual/hi/oa_pilot/h2020-hi-oa-pilot-guide_en.pdf

ESPAÑA. Jefatura del Estado. (2011). Ley 14/2011, de 1 de junio, de la Ciencia, la Tecnología y la Innovación. BOE, 131, 54387-54455 [consulta: 4-10-2014]. Disponible en: http://www.boe.es/boe/dias/2011/06/02/pdfs/BOE-A-20119617.pdf

ESPAÑA. Jefatura del Estado (2014). Ley 21/2014, de 4 de noviembre, por la que se modifica el texto refundido de la Ley de Propiedad Intelectual, aprobado por Real Decreto Legislativo 11996, de 12 de abril, y la Ley 1/2000, de 7 de enero, de Enjuiciamiento Civil. BOE, 268, 90404-90439 [consulta: 3-1-2015]. Disponible en: https://www.boe.es/diario_boe/txt.php?id=BOE-A-2014-11404

ESPAÑA. Ministerio de Economía y Competitividad (2013). Plan Estatal de Investigacion Cientifica y Técnica y de Innovación 2013-2016 [consulta: 7-112014]. Disponible en: www.idi.mineco.gob.es/stfls/MICINN/Investigacion /FICHEROS/Plan_Estatal_Inves_cientifica_tecnica_innovacion.pdf

ESPAÑA. Secretaría de Estado de Investigación, Desarrollo e Innovación (2015). Resolución de 17 de junio de 2015, de la Secretaría de Estado de Investigación, Desarrollo e Innovación, por la que se aprueba la convocatoria para el año 2015 del procedimiento de concesión de ayudas correspondientes al Programa Estatal de Investigación, Desarrollo e Innovación Orientada a los Retos de la Sociedad, en el marco del Plan Estatal de Investigación Científica y Técnica y de Innovación 2013-2016.BOE, 149, 52318-52367 [consulta 19-9-2015]. Disponible en: https://www.boe.es/boe/dias/2015/06/23/pdfs/BOE-A-20156984.pdf

FECYT (2014). Recomendaciones para la implementación del artículo 37: difusión en acceso abierto de la Ley de la Ciencia, la Tecnología y la 
Innovación [consulta: 11-1-2015]. Disponible en: http://www.fecyt.es/fecyt/censoPublicacion.do?

FIAB (2011). Declaración de la IFLA sobre el acceso abierto : definición de su posición y política [consulta. 1-11-2014]. Disponible en: http://www.ifla.org/ files/assets/hq/news/documents/ifla-statement-on-open-access-es.pdf

GALLO, G. (2013). "Cambios en la directiva de reutilización". Blog Entre Códigos Civiles Y Androides.

HARNAD, S. (2005). "The implementation of the Berlin Declaration on Open Access: report on the Berlin 3 Meeting held 28 February-1 March 2005, Southampton".UK. D-Lib Magazine, 11 (3) [consulta: 26-10-2014]. Disponible en: http://www.dlib.org/dlib/march05/harnad/03harnad.html

KROES, N. (2013). Opening up Scientific Data [consulta: 6-9-2014]. Disponible en: file:///C:/Users/Nestor/Downloads/SPEECH-13-236_EN.pdf

NEYLON, C. (2013). "Public Library of Science's Cameron Neylon on the state of Open Access: Where are we, what still needs to be done?" Open and Shut? [blog] [consulta: 12-11-2014]. Disponible en: www..richardpoynder.co.uk/thestate-of-open-access.html

RAMOS SIMÓN, L. F.; ARQUEROAVILÉS, R.; BOTEZÁN, I.; COBO SERRANO, S.; Sala Jiménez, A.; Sánchez Jiménez, R. y Valle Gastaminza, F. (2012). "De la reutilización de información del sector público a los portales de datos abiertos en Europa". BiD: Textos Universitaris de Biblioteconomia I Documentació, 29 [consulta: 20-6-2014]. Disponible en: http://bid.ub.edu/29/ramos2.htm

RIZOR, S. L., \& Holley, R. P. (2014). "Open Access Goals Revisited: How Green and Gold Open Access Are Meeting (or Not) Their Original Goals" [consulta: 8-2-2015]. Journal of Scholarly Publishing, 45(4), 321-335. Disponible en: doi:10.3138/jsp.45.4.01

SIMMONS College (2014). "Declarations in support of OA" [consulta 13-102014]. Open Access Directory Disponible en: http://oad.simmons.edu/oadwiki/ Declarations_in_support_of_OA

SUBER, P. (2013). Open access overview: focusing on open access to peerreviewed research articles and their preprints [consulta: 30-19-2014]. Disponible en: http://legacy.earlham.edu/ peters/fos/overview.htm

TOROSÁNCHEZ-BLANCO, P. (2009). DRIVER. Directrices para el repositorio institucional del SSPA. Slideshare, 9-11-2009 [consulta: 12-6-2014]. Disponible en: http://es.slideshare.net/bvsspa/driver-directrices-para-elrepositorio-institucional-del-sspa

WORKING Group on Expanding Access to Public Research Findings (2012). Accessibility, sustainability, excellence: how to expand access to research publications [consulta: 14-11-2014]. Disponible en: www.researchinfonet.org/wp-content/uploads/2012/06/Finch-Group-reportFINAL-VERSION.pdf 